\title{
LOS ALEMANES EN EL CARIBE COLOMBIANO. MIGRACIÓN, SOCIABILIDAD Y NACIONALSOCIALISMO EN BARRANQUILLA, 1919-1945
}

\author{
GERMANS IN THE COLOMBIAN CARIBBEAN. MIGRATION, SOCIABILITY \\ AND NATIONAL SOCIALISM IN BARRANQUILLA, 1919-1945 \\ Julián Andrés Lázaro Montes* \\ Universidad de Cartagena, Cartagena de Indias, Colombia
}

\begin{abstract}
RESUMEN: El presente artículo explora las dinámicas de migración y sociabilidad de los inmigrantes alemanes en la ciudad de Barranquilla, en el Caribe colombiano, durante un periodo que comprende la posguerra y la segunda guerra mundial. A través del análisis de distintas fuentes, como prensa, informes de inteligencia del gobierno colombiano y documentos generados por los mismos inmigrantes, entre ellos algunas publicaciones seriadas, se analiza la manera como estos extranjeros desplegaron toda una serie de dispositivos de sociabilidad que les permitieron una sólida conexión con la sociedad de acogida, y entre sí y su lugar de origen, elementos que estuvieron en la base del éxito de muchas de sus iniciativas empresariales y que los convirtieron en protagonistas de primer orden del desarrollo económico del puerto caribeño en la primera mitad del siglo XX. Se explora además el efecto de la llegada del Nacionalsocialismo a este grupo de inmigrantes, y la manera como los procesos políticos internacionales de finales de la década de los treinta y años posteriores, determinaron una profunda transformación en las condiciones de vida de estos extranjeros en el Caribe colombiano.
\end{abstract}

PALABRAS CLAVE. Inmigrantes alemanes, Barranquilla, Caribe colombiano, nacionalsocialismo, sociabilidad, segunda guerra mundial.

ABSTRACT: This paper explores the dynamics of migration and sociability of the German immigrants in the city of Barranquilla, in the Colombian Caribbean, during a period that includes the postwar period and the Second World War. Through the analysis of alternative sources, such as the press, intelligence reports of the Colombian government and documents generated by the immigrants themselves, among them serial publications, the way in which these foreigners deployed a series of sociability devices that allowed a connection with the host society, and with each other and their place of origin, elements that are the basis of the success of many business initiatives and that have become the leading players in the economic development of the Caribbean port in the first half of the 20th century. The effect of the arrival of National Socialism on this group of immigrants was also explored, and the way in which the international processes of the end of the 1930s and later years determined a profound transformation in the living conditions of these foreigners in the Caribbean Colombian.

KEYWORDS: German Inmigrants, Barranquilla, Colombian Caribbean, National Socialism, Sociability, Second World War.

* Correspondencia a: Julián Andrés Lázaro Montes. Instituto Internacional de Estudios del Caribe, Universidad de Cartagena, Carrera 4 No. 38-40 Claustro la Merced, Código Postal: 130001 - julianandreslazaro@gmail.com - https://orcid. org/0000-0001-6559-8283

Cómo citar: Lázaro Montes, Julián Andrés (2020). «Los alemanes en el Caribe colombiano. Migración, sociabilidad y Nacionalsocialismo en Barranquilla, 1919-1945»; Historia Contemporánea, 62, 49-77. (https://doi.org/10.1387/hc.20129).

Recibido: 10 agosto, 2018; aceptado: 20 julio, 2019.

ISSN 1130-2402 - eISSN 2340-0277 / (C) 2020 UPV/EHU

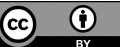

Esta obra está bajo una licencia

Creative Commons Atribución 4.0 Internacional 


\section{Introducción}

Entre finales del siglo XIX y los primeros años del XX se asentaron en la ciudad caribeña de Barranquilla, en el norte de Colombia, un grupo no numeroso de inmigrantes alemanes que se destacó por su participación en el proceso de desarrollo económico que vivió dicha población ${ }^{1}$ hasta bien entrada la década de $1930^{2}$. La constitución, consolidación y expansión de firmas comerciales, la puesta en marcha de empresas de navegación fluvial y aérea, la prestación de servicios financieros, el impulso al desarrollo de industrias, y otras tantas actividades económicas, muchas de ellas exitosas, con que se ha caracterizado a este grupo de inmigrantes, tiende a asociarse en las representaciones que sobre ellos se han construido en el imaginario popular con ciertos rasgos considerados como propios o típicos del ethos alemán, entre ellos la tenacidad, la disciplina y el orden ${ }^{3}$.

Este tipo de imágenes elementales y en buena medida superficiales que se construyen en torno a ciertos individuos o grupos de personas especialmente representativos o con algún rol de importancia en el desarrollo de determinados procesos históricos, tiende a difundirse y consolidarse entre la opinión pública, contribuyendo más a la simplificación de realidades complejas que a la comprensión integral del pasado. Así las cosas, eventos y procesos que deben ser considerados atendiendo a múltiples factores que los hacen posibles, terminan siendo representados a través de narrativas compuestas por explicaciones unicausales, que ocultan mucho más de lo que cuentan y que contribuyen a la supervivencia de formas de historia más cercanas al mito que a la real complejidad de los fenómenos.

La participación en el proceso de desarrollo de Barranquilla de varias de las empresas constituidas y/o impulsadas por esos inmigrantes alemanes es un hecho conocido y documentado. Sin embargo, otros ámbitos de su presencia en la población caribeña que hasta cierto punto explican la dinámica de sus iniciativas empresariales y que también aportan referen-

${ }^{1}$ Para un acercamiento inicial a la historia de Barranquilla se recomiendan los trabajos de algunos autores, entre ellos: Posada Carbó, 1998, 1987; Flórez y Llanos, 1995; Solano y Conde, 1993, Viloria de la Hoz, 2014.

2 Lázaro, 2012.

3 Algunos autores, aunque no muchos, varios de ellos historiadores, otros no, se han ocupado de la presencia y actividades de inmigrantes alemanes en otras regiones de Colombia en diferentes momentos de la historia nacional, entre ellos Rodrigo de Jesús García (1993), Sánchez (1999), De Bedout (1993), Rodríguez Plata (1993) y Martínez (1993). 
cias sobre la historia de la ciudad y de la región del Caribe colombiano, han sido poco estudiados. El presente trabajo constituye precisamente una forma de aproximación al ámbito de las actividades sociales de estos inmigrantes, en un periodo que va desde los primeros años de la primera posguerra del siglo Xx hasta los últimos de la segunda guerra mundial, entendidas dichas actividades como el conjunto de vías a través de las cuales se relacionaron en su condición de advenedizos con la sociedad de acogida, las formas de integración entre sí como miembros de un colectivo particularmente diferenciado y los diferentes espacios, mecanismos y circunstancias que hicieron posible su articulación con el resto de la población en Barranquilla.

La delimitación temporal establecida para el análisis de las actividades sociales de los alemanes en Barranquilla se explica fundamentalmente a partir de dos aspectos de la presencia de estos extranjeros en el Caribe colombiano. En primer lugar, la primera posguerra fue un momento tanto de retornos como de nuevas experiencias de migración, puesto que fue en ese entonces cuando varios de los alemanes que se habían ido de Barranquilla durante el conflicto mundial decidieron volver, en tanto que otros apenas llegaban en su primer o uno de sus primeros viajes a Colombia, dando lugar a una reactivación y también a la puesta en ejecución de prácticas de sociabilidad que les permitieran instalarse y prosperar en su nuevo lugar de permanencia. Por otro lado, la escogencia de los años de la segunda guerra mundial como el otro límite temporal tiene que ver con el hecho de que fue durante el conflicto cuando la actividad social (y de cualquier otro tipo) de estos extranjeros se vio fuertemente condicionada y al final anulada por las medidas de seguridad implementadas a escala continental, por lo que para el caso de Barranquilla (y de otros tantos lugares) se puede considerar como un momento de ruptura de las conexiones que habían logrado establecer en los años anteriores.

El análisis se desarrolla a lo largo de los siguientes cinco apartados. El primero de ellos, el dos en la numeración estructural de este artículo, presenta algunas referencias generales sobre el concepto de marco comunitario de la migración, aplicadas luego al caso específico de los alemanes en Barranquilla para tratar de explicar los factores que motivaron su desplazamiento y permanencia en Colombia. El siguiente apartado, el número tres, se enfoca en la participación de aviadores, técnicos y administrativos alemanes en la guerra entre Perú y Colombia a principios de la década del treinta apoyando las operaciones aéreas colombianas, muchos de ellos re- 
sidentes o conocidos en Barranquilla, lo que representó para la comunidad de alemanes en la ciudad un fortalecimiento de su imagen pública.

El cuatro y el cinco apartados hacen mención de algunas características, prácticas y espacios a través de los cuales los inmigrantes alemanes en Barranquilla se relacionaban entre sí y con la sociedad de acogida. Matrimonios, relaciones laborales, asuntos comerciales y encuentros en espacios de sociabilidad, por mencionar algunas prácticas y mecanismos de contacto, fueron recursos para la construcción de un entorno favorable para la presencia de estos inmigrantes.

Finalmente, el sexto apartado se ocupa de explorar la llegada de ideas nacionalsocialistas al grupo de alemanes en Barranquilla y la forma como la adopción, por lo menos parcial, de las mismas pudo haber incidido en la dinámica de sus relaciones con la sociedad local, sobre todo teniendo en cuenta la radicalidad de los postulados nazis en lo que respecta a la segregación racial y la dificultad de su implementación en las circunstancias propias de los alemanes en el Caribe colombiano.

\section{Marco comunitario de la migración: estímulos y condiciones de inmigración de los alemanes en Barranquilla}

Los procesos de migración constituyen un momento de gran importancia y muy común en la vida de los seres humanos. Difícilmente puede existir una persona que no se haya visto en la situación de cambiar de lugar de residencia y tener que adaptarse a nuevas circunstancias, no siempre favorables, pero a las que debe terminar ajustándose para poder continuar con su trayectoria vital. Estos procesos de migración no se explican solamente a través de la acción de moverse de un sitio a otro, sino que están asociados con y se explican a partir de lo que se denomina marco comunitario de la migración, constituido, entre otros elementos, por el entorno, las circunstancias de momento y las motivaciones para migrar, y que opera en distintos niveles (familia, barrio, ciudad, etc.) como un factor que estimula u obstaculiza la acción de movilizarse ${ }^{4}$.

Como complemento a lo anterior es importante mencionar que el marco comunitario de migración a que se hace referencia no está limitado por las fronteras políticas, sino que se erige por encima de ellas e

4 Jiménez, 2006, pp. 115-118. 
incluye a las sociedades de salida y de llegada, y se amplía o se reduce en función de los factores que lo integran. A manera de ejemplo, se puede señalar que una política específica de un gobierno en materia de estímulo a la migración puede ser parte de ese marco, pero también lo es el conjunto de vecinos y familiares que respaldan o motivan la acción de movilizarse.

Para el caso específico de la presencia alemana entre las décadas del veinte y la del cuarenta en la ciudad portuaria de Barranquilla, es importante señalar que la interpretación del fenómeno a la luz del concepto de marco comunitario debe considerar la situación de Alemania después de la primera guerra mundial, marcada por una profunda crisis que afectó a los distintos ámbitos de la vida de la sociedad y que impulsó la salida de cientos de miles de alemanes del país ${ }^{5}$, algunos de ellos, no muchos en realidad, hacia el Caribe colombiano ${ }^{6}$.

De manera simultánea a la situación en Alemania, operó como factor de atracción en algunos de los inmigrantes alemanes que llegaron hasta el Caribe colombiano la dinámica comercial de una población portuaria como Barranquilla, que desde finales del siglo XIX había venido sirviendo como enlace entre el mercado interno colombiano y los circuitos comerciales internacionales. Para algunos de estos alemanes que se encontraban operando en el Caribe colombiano desde antes de la guerra se trató de reactivar sus negocios, en tanto que para otros, informados por sus conocidos o familiares de las posibilidades que podían llegar a tener o estimulados por los evidentes contactos comerciales entre Barranquilla y las ciudades de Bremen y Hamburgo, se trató de una nueva experiencia en procura de un futuro distinto al que podían atreverse a proyectar en aquel momento en la Alemania de la posguerra ${ }^{7}$.

5 Dallas, 2001; Weitz, 2009; Peukert, 1989; Kitson, 2001.

${ }^{6}$ No existen cifras precisas sobre la cantidad de alemanes que vivían en Barranquilla a finales de los años treinta. Una lista elaborada por el autor de este artículo construida a partir de protocolos notariales, publicaciones seriadas, publicidad comercial y otros documentos que datan de la década del treinta dio como resultado un total de 235 personas de nacionalidad alemana, a los que se deben sumar los hijos de algunos de ellos y, también, en algunos casos, sus esposas venidas de Alemania, aunque es preciso considerar que este grupo de inmigrantes no constituyó una colonia cerrada, sino, más bien, un conjunto de individuos que interactuaban y se relacionaba de manera estrecha con la sociedad de acogida. Para el momento del inicio de la guerra el número de alemanes en Barranquilla probablemente no fuera superior a 500 .

7 Washausen, 1968. 
Con el propósito de avanzar en la exploración de las relaciones sociales de los alemanes en Barranquilla, es importante entrar a considerar ciertos elementos del marco comunitario de la migración asociados al contexto del Caribe colombiano y de manera específica a la sociedad de acogida, los cuales hicieron posible el arribo y consolidación de la presencia de estos inmigrantes.

En primer lugar, la presencia de inmigrantes alemanes después de la Gran Guerra contó con un precedente que de alguna forma ayudaba a configurar un imaginario altamente favorable para este tipo de extranjeros. Además de las empresas que venían operando en la ciudad de propiedad o con participación de alemanes, como las diferentes iniciativas empresariales de Adolf Held, por mencionar un caso representativo, ${ }^{8}$ estaba el no menos importante hecho de la participación de una firma alemana, la Hoenisberg \& Wessels, en la construcción del Ferrocarril de Bolívar, obra de suma importancia para el despegue comercial que vivió la ciudad desde principios de la década de 1870, logrando articular el puerto marítimo, ubicado en la vecina población de Sabanilla, con el puerto fluvial de Barranquilla9.

De la particular buena disposición que existía en, por lo menos, una parte de la población de Barranquilla hacia estos inmigrantes y que se constituía como parte de un marco favorable para la inmigración y el establecimiento de ellos da cuenta un anuncio publicado en un periódico de la ciudad en 1905: «Ha partido para Alemania el señor D. Adolf Ringe, quien ha residido varios años entre nosotros. Como es sabido, la colonia alemana se distingue por su laboriosidad, honradez y aptitudes especiales para asimilarse nuestros hábitos y costumbres. De ahí que cuando alguno de sus miembros parte, los colombianos sentimos la separación como si se trata de un compatriota» ${ }^{10}$.

A las percepciones derivadas del trato cotidiano con estos inmigrantes y que se materializaban, entre otras formas, a través de muestras como la nota citada, muy seguramente les acompañaban las ideas acerca de pureza racial que se difundieron en Colombia entre finales del siglo XIX y las primeras décadas del xx, y que debieron contribuir con la generación de un clima bastante favorable principalmente para inmigrantes europeos (sobre

${ }^{8}$ Meisel y Viloria, 1999.

9 Nicholls, 1973.

10 Archivo Histórico del Atlántico (en adelante A.H.A.), El Promotor, mayo 13 de 1905, p. 2. 
todo del norte de Europa) y norteamericanos. Un reconocido político conservador como Miguel Antonio Caro había expresado hacia 1867 la idea de que «los pueblos se civilizan recibiendo ciencia y costumbres de otros mejor dotados» ${ }^{11}$, y en la misma línea se expresaban varias décadas después, ya bien entrado el siglo $\mathrm{xx}$, múltiples opiniones al respecto, como las del también político Luis López de Meza, quien llegó a ser ministro en las carteras de Educación y Relaciones Exteriores, defensor de la idea de que la mezcla racial de elementos europeos con nativos de América y esclavos africanos había sido generadora en sus descendientes de «trastornos de carácter», «delincuencia», e «inadaptación social» ${ }^{12}$.

En correspondencia con lo anterior, ciertos sectores de la opinión pública barranquillera adhirieron a esa visión, tal y como lo evidencia una nota del diario local La Prensa del año 1933, que hacía referencia a la necesidad de «llevar a cabo [en Colombia] una colonización de razas seleccionadas, como la alemana, la americana, la francesa, etc..$^{13}$.

A la creación de un marco favorable para la migración alemana y la inserción en la sociedad local de estos inmigrantes, ya en la década de 1920, contribuyeron algunos gestos y acciones que pueden interpretarse como formas de acercamiento a elementos de cierto valor simbólico para los colombianos. Por ejemplo, varias de las aeronaves de la Sociedad Colombo-Alemana de Transportes Aéreos, SCADTA, fundada a finales del 1919 y con una considerable presencia germana, tomaron nombres asociados con la geografía colombiana, de ciudades o departamentos, como sucedió con los aviones «Tolima», «Cúcuta» y «Atlántico». En la misma línea de lo anterior, la empresa de navegación fluvial H. Lindemeyer \& Cía., con fuerte presencia accionaria de alemanes, bautizó a varios de sus vapores con nombres de gran resonancia para los colombianos: «Tenerife», población ubicada en las ribera del Río Magdalena; «Bocas de Ceniza», nombre de la desembocadura del mencionado río en el Caribe, cerca de Barranquilla; "García Rovira», nombre de caudillo regional en los procesos de independencia; «Cartagena», el nombre de una de las ciudades más representativas del Caribe colombiano.

El marco comunitario de migración a que se ha hecho referencia también incluía, como ya se sugirió, a las relaciones interpersonales como re-

11 Caro, citado en Hering, 2018, p. 75.

12 López, 1970.

13 A.H.A, «Mil familias alemanas en el Amazonas», La Prensa, 8 de marzo de 1933, p. 8 . 
curso para estimular la movilización y lograr la inserción en el medio local. Un caso en especial puede aportar algunas referencias al respecto.

Hacia el año de 1923 y muy probablemente buscando distanciarse de la crítica situación en que se encontraba Alemania, llegó hasta Barranquilla Walter Ritzel ${ }^{14}$. El puerto colombiano, aunque bien conectado con Europa, no alcanzaba las dimensiones ni presentaba el atractivo de otros complejos portuarios del continente americano, por lo que la llegada de individuos como Ritzel se puede explicar más desde las redes y las relaciones interpersonales. De hecho, varias de las empresas alemanas que operaban en Barranquilla habían estimulado la movilización de ciudadanos alemanes a los que vinculaban en sus actividades, en ocasiones ampliándolas a otras ciudades de Colombia.

Las conexiones con los alemanes ya residentes en Barranquilla y con las organizaciones que ellos constituían representaban un recurso para la inserción de los inmigrantes en la sociedad local. A Ritzel se le puede ubicar ya en 1927 como parte de la Junta Directiva del Colegio Alemán en Barranquilla, en el cargo de Vocal, proyectándose años más tarde como vicepresidente (1932-1933) y como presidente (1934-35 y 1936-37) de la misma organización ${ }^{15}$. En cuanto a la inserción de Ritzel en la vida económica de la ciudad, posible a través de las mencionadas redes interpersonales, constitutivas del marco comunitario para la migración, hay referencias que lo ubican ingresando junto con otro alemán, Werner Backhaus, como socio de la Empresa Hanseática Wiese \& Starck, firma en la que se encontraban otros empresarios alemanes y que se dedicaba a la elaboración de artículos de uso doméstico, como velas y jabones, y al transporte de mercancías por el río Magdalena ${ }^{16}$. También aparece vinculado una década después con la Compañía de Industrias Nacionales, acompañando a otros empresarios, también alemanes, en la producción de insumos para la construcción de viviendas y artículos asociados ${ }^{17}$.

14 Archivo General de la Nación/Archivo Ministerio de Relaciones Exteriores (en adelante A.G.N./A.M.R.E.), Fondo Diplomática y Consular, Caja 23, Carpeta 18, «Informe de la Sección de Extranjería e Identificación de la Policía Nacional, en Barranquilla, al Director General», 23 de mayo de 1943, folio 76.

15 Lobo y Lober-Sies, 1994, p. 20.

16 Meisel y Viloria, 1999.

17 A. H. A., Notaría Primera de Barranquilla, E.P. 660, 24 de mayo de 1938, «Ratificación de venta: Fritz Fuhrhop a favor de Compañía de Industrias Nacionales». 
Un marco comunitario de migración puede ser muy amplio e incluir a tantos elementos como se quiera en la medida en que se les pueda considerar como factores que estimulan la migración y contribuyen con la inserción de los recién llegados en la sociedad de acogida. Los señalados hasta ahora pueden considerarse como básicos, teniendo en cuenta que hacen referencia a antecedentes de migración, imagen favorable sobre la misma y existencia de redes interpersonales que trascienden fronteras políticas y que contribuyen a motivar la movilidad y hacen menos difícil la situación del inmigrante en su nuevo lugar de residencia. Sin embargo, para los inmigrantes alemanes en Barranquilla constituyeron apenas un punto de partida, unas condiciones iniciales a partir de las cuales fueron construyendo unas formas de relación con la sociedad local que tuvieron diversas formas de materialización, tal y como se verá en las siguientes páginas.

\section{La guerra colombo-peruana como coyuntura favorable para la integración con la sociedad de acogida}

A comienzos de septiembre de 1932 un grupo de peruanos armados invadió por su propia cuenta algunas poblaciones del Amazonas colombiano. La acción fue respaldada poco después por el gobierno del Perú, con lo que un incidente protagonizado por unos invasores terminó por transformarse en un conflicto entre los dos países ${ }^{18}$.

La situación se presentó particularmente compleja para el gobierno colombiano, puesto que fue un conflicto inesperado y en una zona de difícil acceso para las tropas, algunas de las cuales sólo pudieron ser desplegadas en el teatro de operaciones ingresando luego de largas jornadas a través de vías fluviales. Frente a esta situación, la aviación se presentó como una gran alternativa para poder responder a la agresión de los peruanos, por lo que el presidente Enrique Olaya Herrera no dudó en recurrir a la Sociedad Colombo-Alemana de Transportes Aéreos (SCADTA) para que pusiera a disposición de las Fuerzas Armadas al personal de la compañía, integrado por una buena cantidad de técnicos y pilotos alemanes, varios de estos últimos poseedores de una experiencia importante en batalla debido a su participación en la primera guerra mundial ${ }^{19}$.

\footnotetext{
18 Pinzón, 1990.

19 Boy, 1963, pp. 166-170.
} 
La presencia del personal alemán en las operaciones fue muy bien recibida en Barranquilla. Las razones de ello están asociadas al hecho de que la SCADTA había sido creada en la ciudad caribeña y una buena cantidad de sus operaciones se desarrollaban desde allí. Aviadores, técnicos y funcionarios administrativos, varios de los cuales habían recibido responsabilidades asociadas con la política de defensa nacional en ese momento de crisis, eran ampliamente conocidos en la sociedad local, vistos ya como parte de la misma. A esto se sumaba la percepción de que no sólo estos individuos sino también otras empresas alemanas estaban contribuyendo con el esfuerzo de guerra desplegado por Colombia, lo que se evidenciaba, según algunos diarios locales, en la llegada de más pilotos alemanes y en el suministro de armas y municiones traídos por empresas marítimas de Alemania ${ }^{20}$.

Las manifestaciones de reconocimiento y admiración por los aviadores alemanes ocupaban considerables espacios en medios de comunicación locales. Pedro Pineda Vélez, un periodista del diario La Prensa, se explayaba en elogios al referirse a Herbert Boy, comandante de las operaciones de la flota colombiana, de quien decía que «con cien colombianos como Boy ya el Perú estuviera barrido de filibusteros». Y sobre el aviador alemán afirmaba también que «en tecnicismo aéreo es un decisivo elemento de combate: díganlo si no las bajas peruanas, los triunfos de Boy verificados en poco tiempo [...] que los aviadores peruanos [ante] su presencia corren presurosos» ${ }^{21}$.

Otras manifestaciones de exaltación y homenaje también se registraron en Barranquilla, algunas particularmente llamativas. Por ejemplo, estuvo la de la firma comercial Mora Orama y Cía., que abrió una fábrica de calzado con dos líneas cuyos nombres hacían referencia a los sucesos de la guerra con el Perú, una de ellas exclusiva para mujeres llamada «Leticia», en clara referencia al nombre de la población colombiana que había caído en manos de los peruanos, en tanto que la otra línea, la de caballeros, llamada «Boy», en homenaje al piloto alemán ${ }^{22}$.

Pero no sólo Herbert Boy se llevaba los reconocimientos, otros tantos aviadores alemanes se erigieron como héroes en la medida en que los

20 A.H.A., «A bordo del "Ingrid Horn" debieron llegar a Puerto Colombia ocho aviadores alemanes», La Prensa, 31 de marzo de 1933, p. 1a.

21 Pineda, 1933.

22 A.H.A., «Una fábrica de calzado marca "Leticia” y "Boy”», La Prensa, 21 de marzo de 1933, p. 8a. 
medios de comunicación iban dando cuenta de sus aventuras en el sur del país. Las circunstancias especialmente difíciles que debieron enfrentar estos extranjeros en un entorno de difícil acceso y complejidad como el Amazonas constituían el insumo a partir del cual se elaboran representaciones que era difundidas en la sociedad barranquillera de la época. El diario La Prensa describía en marzo de 1933 lo sucedido al piloto Hans Himpe, a quien «lo sorprendió una fuerte tempestad, contra la cual tuvo que luchar varias horas [...] Valientemente se enfrentó el aviador a las adversas condiciones atmosféricas, ejecutando todas las maniobras que su experiencia le aconsejaba» ${ }^{23}$.

Las situaciones trágicas también sirvieron para acercar estos inmigrantes a la sociedad local. A mediados de abril de ese año de 1933 se llevaron a cabo en Barranquilla las honras fúnebres de Herbert Grosmann, mecánico de aviación que había fallecido luego de varios días de convalecencia en un hospital de la ciudad debido a una enfermedad contraída en la zona de combates del sur colombiano. En el acto se hicieron presentes las autoridades locales y departamentales, tanto civiles como militares, además de una buena cantidad de personas del común, según reportaba un diario local: «También estuvo presente en el desfile la Asociación de la Mujer Colombiana [...] Cuando el desfile se aproximaba al cementerio, voló sobre la concurrencia un avión del gobierno nacional, adornado con banderas negras [...] Durante el recorrido hubo momentos en que el desfile llenaba más de dos cuadras, tal afluencia de público perteneciente a todas las categorías sociales» ${ }^{24}$.

La circulación de información sobre estos inmigrantes y su participación en las acciones de defensa de la soberanía colombiana implicaba también la difusión de rasgos biográficos, los cuales muchas veces daban cuenta de los lugares de procedencia en su país de origen de estos pilotos, técnicos y hombres de negocios, con lo que la imagen sobre Alemania y algunas de sus ciudades se hacía especialmente positiva, máxime si se tiene en cuenta los nexos comerciales de Barranquilla con algunos puertos alemanes. Sucedía así con el caso del Capitán Behrend, fallecido también por una enfermedad contraída en operaciones, de quien un diario local en uno de sus artículos afirmaba que era oriundo

23 A.H.A., «Un aviador alemán estuvo perdido tres días en medio de indios», La Prensa,16 de marzo de 1933, p. 9a.

24 A.H.A., «Estuvo sumamente concurrido el entierro del mecánico de aviación señor Herbert Grossmann», La Prensa, 15 de abril de 1933, p. 8a. 
de «la dulce y acogedora ciudad de Hamburgo, [donde] un torrente de lágrimas correría por el héroe ausente, por el hijo mimado que inquieto y lleno de idealismo como todos los hijos de la Germania inmortal se lanzó hacia nuestras tierras, solamente con el noble deseo de pelear [...] por las causas justas» ${ }^{25}$.

De entre los mismos alemanes en Barranquilla surgieron manifestaciones de respaldo a la labor de sus compatriotas en el conflicto con el Perú, algunas de las cuales adquirieron tintes de reivindicación de participación germana no reconocida por la historia de Colombia, como puede apreciarse en un escrito publicado por Albert Tietjen en un diario local en el que recordaba los casos del Coronel Uzlar y de Otón Felipe Braun, ambos participes de los procesos de independencia de la Nueva Granada, remarcando las cercanía de Colombia con un pueblo, el alemán, con el que «siempre ha llevado inalterables relaciones de una buena $\operatorname{amistad} \gg^{26}$.

El conflicto colombo-peruano contribuyó entonces a fortalecer las ya de por sí bastante positivas relaciones que sostenían los inmigrantes alemanes con, por lo menos, una buena parte de la sociedad local. Al imaginario de los inmigrantes como agentes dinamizadores del progreso local y regional se sumó en este contexto la idea de que eran además grandes protagonistas de la defensa de la soberanía nacional, todo lo cual, en su conjunto, constituyó un marco favorable para la consolidación de estos extranjeros como parte de la elite social y económica de la Barranquilla de la época.

\section{Los alemanes y sus formas de relación con la sociedad de acogida}

La mencionada integración en la sociedad local tuvo diversas formas, una de ellas asociada al ingreso a círculos exclusivos y de especial relevancia en términos económicos y políticos en la ciudad. Un caso representativo de ello fue el de la ya referenciada SCADTA, empresa de aviación en cuya creación, en el año de 1919, convergieron los intereses y las

25 A.H.A., «Discursos del ministro alemán y el teniente Escandón en el entierro del Capitán Behrend», La Prensa, 18 de marzo de 1933, p. 8a.

26 A.H.A., «Los alemanes en la guerra magna», La Prensa, 20 de abril de 1933, p. 7a. 
iniciativas de hombres de empresa alemanes ${ }^{27}$ y nativos ${ }^{28}$. También se puede mencionar el caso de la Empresa de Vapores Lindemeyer, encargada del transporte por el río Magdalena y otros cauces de agua, que a comienzos de los años treinta contaba entre sus accionistas (aunque en una proporción no mayoritaria) a algunas personas de Barranquilla ${ }^{29}$.

En lo que respecta a la cercanía con algunas familias destacadas de la ciudad, se puede mencionar el caso de Albert Lühr, socio de una importante casa comercial alemana, la Breuer, Möller \& Co., quien en el año de 1912 contrajo matrimonio con la señorita Hortencia Carbonell, integrante de una notable familia de la ciudad entre cuyos miembros estaba Abel Carbonell, tiempo después Alcalde de Barranquilla y con cierta figuración a nivel nacional cuando ocupó la Secretaría del Ministerio de Comunicaciones de Colombia.

La revisión del testamento de Lühr, presentado ante notario público a principios de los años veinte lo muestra para ese momento como un destacado empresario de la ciudad, al que sirven como albaceas testamentarias dos compatriotas suyos, también empresarios, Richard Schraeder y Alfred Rehbein ${ }^{30}$. Esta situación de evidente cercanía con individuos con los que compartía el lugar de procedencia revela también otro rasgo de las actividades sociales de estos inmigrantes: la tendencia a crear y consolidar vínculos entre sí de manera simultánea a los que sostenían con los locales.

Otro caso que guarda ciertas similitudes con el anterior, aunque en un nivel social menos visible es el de Franz Friedrich Kurt Richels, técnico mecánico que llegó a Barranquilla en el año de 1930 y que se casó con la señora Aura Buitrago, hermana del Gerente de la Central de Loterías del Atlántico. Al parecer la vida de Richels fue tranquila y menos llamativa que la de otros alemanes, manteniendo incluso cierta distancia con

27 Entre el grupo de fundadores de la SCADTA aparecen los alemanes Albert Tietjen, Stuart Hosie y Werner Kaemmerer, ya conocidos e integrados en la sociedad local, a los que se sumó poco después técnicos y aviadores que en poco tiempo establecieron fuertes conexiones con el entorno local, de entre los cuales se destacaron en la historia de la compañía Fritz Hammer, Wilhelm Schnurbusch, Herbert Boy y Helmuth Von Krohn.

28 Lázaro, 2014.

29 En la Empresa de Vapores Lindemeyer el accionista mayoritario era el alemán Heinrich Lindeyemer, que poseía algo más del 50\% del total de la compañía, en tanto que el otro $50 \%$ se repartía entre socios colombianos y alemanes. A.H.A., Notaría Segunda de Barranquilla, E.P. 544, 26 de febrero de 1930.

30 A.H.A., Notaría Primera de Barranquilla, E.P. 1822, 21 de agosto de 1920, «Testamento abierto hecho por August Albert Johannes Lühr», folios 272-273. 
respecto a las organizaciones adeptas al Nacionalsocialismo que se empezaron a hacer notar desde mediados de la década de treinta. Richels no despertó sospechas en los organismos de seguridad colombianos cuando éstos empezaron a hacer seguimiento sobre los alemanes en Barranquilla, e incluso se hace referencia en un informe de dichos organismos a que, además de ser un hombre confiable, era respaldado por su cuñado, alguien intachable y de muy buena reputación en la ciudad ${ }^{31}$.

Finalmente, un par de casos más aportan algunas referencias adicionales y completan un cuadro más o menos aproximado a lo que fueron las relaciones de estos inmigrantes con la sociedad local. Franz Copell era oriundo de Braunau, y había iniciado una relación desde 1919 con una mujer de origen rural, Raquel Mendoza, con quien tuvo tres hijos, Carmen, Andrea y Francisco, en 1920, 1923 y 1925, respectivamente, los cuales fueron reconocidos como tales ante notario público en el año de 1926. Luego de esta diligencia, Copell procedió a declarar a sus recién reconocidos hijos como herederos de unos terrenos rurales y unas «cabezas de ganado» ${ }^{32}$. En los trámites mencionados aparece como testigo legal un conocido suyo (probablemente cercano) llamado Julio Vargas. Todo lo anterior evidencia algunos elementos que difieren notablemente del imaginario construido sobre los alemanes en la ciudad: uno, la informalidad en que mantuvo la relación con esposa e hijos; dos, la orientación de este individuo hacia las actividades agrícolas. Llama también la atención que el testigo de las gestiones legales de Copell no sea un alemán, situación bastante atípica y que puede ser un pequeño indicio que apunte hacia una relación más estrecha con los nativos que con sus coterráneos, por fuera de los círculos empresariales en que se insertaban otros alemanes.

El otro caso final es el de Eduard Asmus, un técnico cervecero alemán que desde el año 1920 se encontraba en el Caribe colombiano, laborando primero en la Cervecería Bavaria y luego, desde 1936, como vigilante en la Compañía Algodonera de la Costa Atlántica. Estaba casado con una mujer de la región y tenía seis hijos. Vivía con su familia en una población semirrural cercana a Barranquilla, donde tenía una pequeña finca con algunas reses y otros animales de crianza. Asmus también estaba al margen de

31 A.G.N./A.M.R.E., Diplomática y Consular, Caja 23, Carpeta 181, «Informe de la Sección de Extranjería e Identificación de la Policía Nacional», 23 de mayo de 1943, folios 78-79.

32 A.H.A., Notaría Primera de Barranquilla, E.P. 1147, 21 de junio de 1926, «Reconocimiento de varios hijos naturales que hace Franz Copell». 
ese grupo de empresarios con el que se asocia con frecuencia a la presencia alemana en Barranquilla, mucho más cercano a la sociedad de su entorno inmediato, que era fundamentalmente rural y nativa. Cuando al Gerente de la Compañía Algodonera se le preguntó por parte de las autoridades colombianas sobre la posible vinculación de Asmus con el grupo nazi local, éste respondió: «es el tipo de campesino alemán que no está muy enterado de política y que tales acontecimientos no le importan para nada» ${ }^{33}$.

Lamentablemente las huellas de esos «otros» alemanes, de los cuales los dos últimos ejemplos constituyen una muestra, son difíciles de rastrear, puesto que por no ser destacados empresarios o figuras públicas de gran visibilidad en la sociedad barranquillera no aparecen con la frecuencia con que sí lo hacen otros paisanos suyos, sin embargo, los registros que dejaron ayudan a poner en evidencia rasgos distintos a los que tradicionalmente han servido como base para construir un imaginario sobre los alemanes en el Caribe colombiano.

De cualquier forma, es preciso retornar sobre esos alemanes que sí dejaron registros a través de los cuales poder seguir sus trayectorias de vida y sus interacciones con el resto de la sociedad local. Precisamente dichas interacciones ocurrían en unos espacios determinados, que cumplían una función aglutinadora de estos inmigrantes entre sí (como se verá en el siguiente apartado), pero que al mismo tiempo servían como escenarios de encuentro con individuos y grupos diferentes.

Uno de esos espacios de sociabilidad era el Club Alemán, donde se llevaron a cabo en múltiples ocasiones fiestas de integración entre alemanes e invitados externos a la comunidad alemana de Barranquilla. A manera de ejemplo encontramos la época de Carnaval de 1939, cuando, en correspondencia con las fiestas que dinamizaban la ciudad para estos primeros meses de cada año, se enviaron desde las directivas del Club las invitaciones a varias familias no alemanas de la ciudad para que tomaran parte en una fiesta que se desarrollaría en las instalaciones del mismo ${ }^{34}$. También aparecen las ceremonias de recepción en el Club de visitantes de la ciudad considerados «ilustres», como sucedió en el año de 1937 con la llegada a Barranquilla del presidente de la República de Colombia, Al-

33 A.G.N./A.M.R.E., Diplomática y Consular, Caja 23, Carpeta 181, «Informe de la Sección de Extranjería e Identificación de la Policía Nacional», 23 de mayo de 1943, folios 82-83.

${ }^{34}$ IberoAmerikanische Institut (en adelante I.A.I) «Anuncio», Karibischer Beobachter, No. 4, 15 de febrero de 1939, p. 22. 
fonso López Pumarejo, y su familia, evento en el que participaron miembros de la elite política y económica de la región ${ }^{35}$.

Otro espacio también utilizado para generar actividades de encuentro entre colombianos, alemanes y personas de otras nacionalidades era el Colegio Alemán. Allí se desarrollaban distintos tipos de eventos, algunos de ellos relacionados con celebraciones de fechas especiales y sobre los cuales es posible encontrar referencias en medios impresos de comunicación. Una de estas referencias nos la proporciona el Karibischer Beobachter, la revista nazi que se editaba y circulaba en Barranquilla, donde en enero de 1937 aparece la mención a las distintas actividades, entre ellas bazares y fiestas, desarrolladas en las celebraciones de Navidad y Año Nuevo de 1936, en las que se contó con una «alegre participación» de colombianos ${ }^{36}$.

Aparecen descritos entonces algunos rasgos que aportan detalles acerca de la manera como los inmigrantes alemanes establecieron relaciones con la sociedad local, no solamente a partir de las conexiones con personas de la elite sino también a un nivel mucho más modesto, y recurriendo en muchas ocasiones al uso de ciertos espacios que operaban como escenarios de sociabilidad en los que se tendían redes y se articulaban con las dinámicas sociales de Barranquilla. Sin embargo, aún entendiendo la importancia de dichas conexiones, los alemanes no dejaron de mantener ciertos espacios como privados, propios y necesarios para fortalecer sus rasgos culturales en un entorno notablemente diferente, espacios donde lo estrictamente alemán como elemento aglutinador y como forma de conexión con su lugar de origen adquiría un papel especial.

\section{La sociabilidad al interior de la comunidad de alemanes en Barranquilla: el Club Alemán y otros espacios de encuentro}

Ya se hizo mención al Colegio Alemán y al Club Alemán como escenarios de encuentro de los alemanes con la sociedad local, por lo menos con una parte de sus miembros, pero ambos espacios tenían en su naturaleza un propósito diferente, como era el de servir de espacio de reunión en torno a elementos que se pueden considerar como estrictamente alemanes.

35 I.A.I, «Agradecimiento», Karibischer Beobachter, No. 2, 15 de enero de 1937, p. 9.

36 I.A.I., «Schulfest 1936», Karibischer Beobachter, No. 2, 15 de enero de 1937, p. 9. 
Luego del retorno de algunos alemanes idos hasta su país durante la Primera Guerra Mundial y con el arribo de otros más en la década del veinte, se reactivaron las actividades de un espacio social como el Club Alemán, que desde 1904 y hasta el inicio del primer gran conflicto del siglo XX sirvió como sitio de encuentro exclusivo (salvo excepciones) para estos extranjeros ${ }^{37}$. La solvencia económica que fueron alcanzando las empresas de estos inmigrantes terminó por impulsar el centro social, y para finales de la década del veinte y los primeros momentos de la del treinta era uno de los más representativos en su tipo de la ciudad.

Efectivamente, el crecimiento que evidenció entre una década y otra hizo necesaria la reformulación de la forma como venía operando el Club. En primer lugar, dejó de funcionar en una casa alquilada y pudo adquirir una propia y más amplia en la cual seguir operando. Por otro lado, se reconfiguró a partir de una figura jurídica que le daba una proyección empresarial, orientada a la prestación de unos servicios a sus socios, funcionando de manera formal con organismos de gobierno interno estables, responsables y sometidos a control, dejando de lado los liderazgos personales basados en la buena voluntad. Estas nuevas condiciones se pusieron en marcha el 17 de marzo de 1930, cuando se constituyó como Sociedad Club Alemán, con un capital inicial de veintidós mil pesos oro americano, fraccionados en un total de 440 acciones $^{38}$.

La reorganización del Club parece haber dado importantes resultados y, como ya se mencionó, el centro social se constituyó como uno de los más representativos de la ciudad, ofreciendo servicios y entretenimiento para los usuarios, entre ellos salones de baile y espacios para la práctica deportiva, algunos de ellos realmente novedosos, como la pista de bolos, la primera que se abrió en la ciudad. Un anuncio de prensa de ese año de 1930 invitaba a una actividad típica de fin de semana en el Club ${ }^{39}$.

\section{LA JUNTA DIRECTIVA DEL CLUB ALEMÁN, S.A. (DEUTSCHER KLUB)}

Se complace en invitar a sus socios a un té danzante que tendrá lugar en los salones del Club el día 9 del presente a las 5.00 p.m.

37 Vos, 1999, p. 36.

38 A.H.A., Notaría Primera de Barranquilla, E.P. 502, 17 de marzo de 1930, «Constitución de una Sociedad Club Alemán».

39 A.H.A., La Junta Directiva, La Prensa, 6 de noviembre de 1930, p. 4a. 
Además se permite avisar que el día sábado 8 del presente a las 4 y media p.m. se jugará la partida final de mixed double.

Se ruega encargar las mesas con anticipación al Sr Admor.

La llegada de los nazis al poder en Alemania en el año de 1933 trajo algunas transformaciones de consideración en la cotidianidad del Club Alemán. El Nacionalsocialismo implementó desde su llegada al gobierno la necesidad de que los alemanes en el exterior se articularan con su proyecto político, lo que se conoce como Gleichschaltung, la alineación o coordinación con el ideario nazi, con lo que aquellos espacios que servían como escenarios de encuentro y sociabilidad de los alemanes dondequiera que estuviesen, como por ejemplo el Club Alemán en Barranquilla, pasaron a ser de especial interés para el gobierno del Reich, que dispuso de sus adeptos y de las autoridades consulares para ejercer efectivo control y orientar las actividades en torno a los propósitos de propaganda y difusión del nazismo.

Esta nueva situación se puso en evidencia apenas unos meses después de la llegada de los nazis al poder en Alemania. En el Club Alemán se desarrolló en abril de 1933 una fiesta para conmemorar el natalicio de Bismarck y el día de la bandera tradicional alemana, ejerciendo como orador central el jefe del grupo nazi local, Erwin Ettel, quien lanzó un discurso acerca de los dos motivos de la fiesta y de la importancia de la llegada del Nacionalsocialismo al gobierno del Reich ${ }^{40}$.

Para el año de 1935 el Club Alemán aparece integrando la Asociación de Clubes Alemanes en el Exterior, organismo que articulaba a los distintos clubes y que definía las políticas que éstos debían seguir para mantenerse alineados con el proyecto nazi ${ }^{41}$. Así las cosas, muchas de las actividades sociales que se desarrollaron a partir de entonces en el Club Alemán de Barranquilla estaban estrechamente relacionadas con los propósitos nacionalsocialistas, como por ejemplo algunos banquetes y fiestas que tenían como objetivo la recolección de fondos económicos para ayudar a los más pobres en Alemania durante la época de invierno ${ }^{42}$.

40 A.H.A., «Una animada fiesta patriótica se celebró el sábado en el Club Alemán», La Prensa, 4 de abril de 1933, p. 3a.

41 I.A.I., «Nachrichten der Deutschen Interessen-Gemeinschaft Barranquilla», Karibischer Beobachter, No. 37, 7 de diciembre de 1935, p. 2.

${ }^{42}$ I.A.I, «Konzert im Deutschen Klub», Karibischer Beobachter, No. 1, 1 de enero de 1938 , p. 14. 
Igualmente, se celebraban fechas importantes dentro del calendario nazi, una de ellas el cumpleaños de Hitler, en la que los salones del Club, decorados de manera especial, recibían a buena parte de la comunidad de alemanes en Barranquilla, que escuchaba los discursos de los líderes nazis locales, buena parte de ellos orientada a resaltar la importancia de la llegada del Führer al poder ${ }^{43}$. También dentro de las fechas del calendario nazi celebradas en Barranquilla por parte de los alemanes en el Club estuvo el 1 de mayo, al que los nazis le dieron un sentido diferente al tradicional, asociado normalmente con el Día del Trabajo, pero que para los alemanes pasó a ser la Jornada Nacional de Alemania.

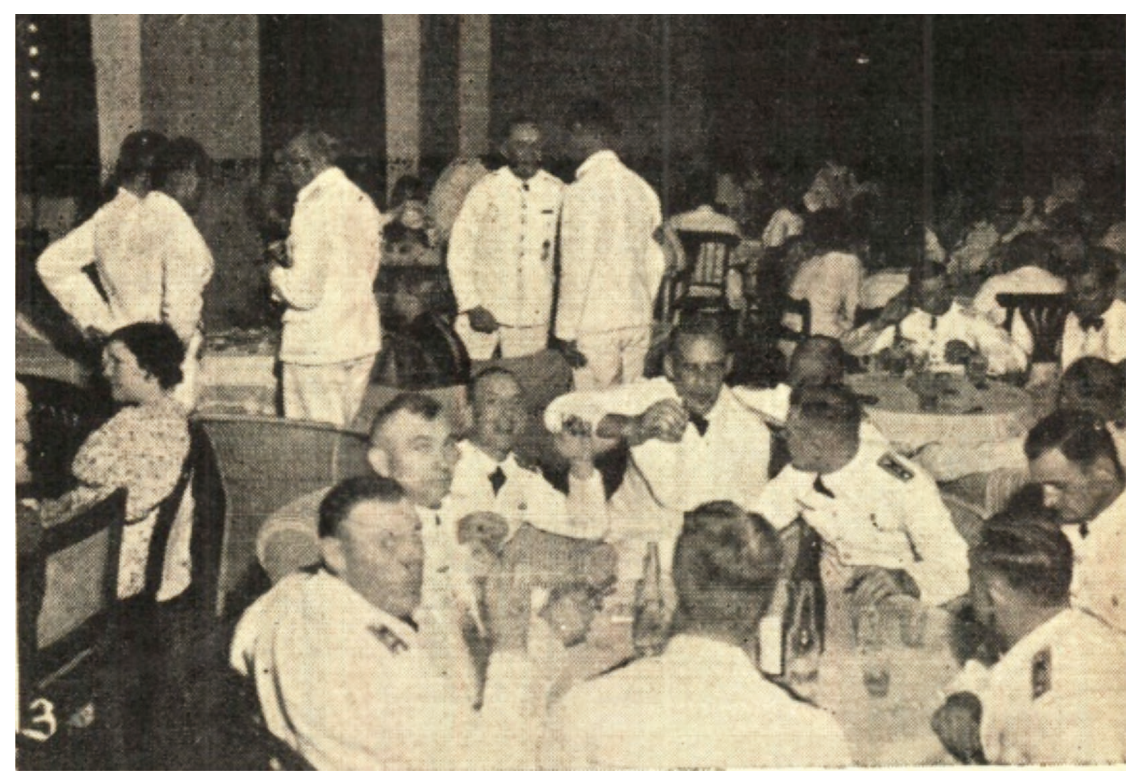

\section{Imagen 1}

Fiesta en el Club Alemán en homenaje a la tripulación del Schleschwig-Holstein, en enero de 1937

Fuente: I.A.I, «Das Linienschiff "Schleswig-Holstein” im Barranquilla», Karibischer Beobachter, No. 3, 1 de febrero de 1937, p. 14.

43 I.A.I, «Feier anlasslich des 50. Geburtstages des Fuehrers im Deutschen Klub», $\mathrm{Ka}$ ribischer Beobachter, No. 9, 1 de mayo de 1939, p.13. 
En ese contexto de agitado nacionalismo que caracterizó a la vida política de los alemanes a finales de los años treinta, especial significado tuvo para estos inmigrantes en Barranquilla la visita de representantes del Estado alemán, tal y como se pudo apreciar en el recibimiento que se dio a comienzos de 1937, cuando el buque de guerra «Schleswig-Holstein» atracó en la vecina población de Puerto Colombia, siendo sus tripulantes trasladados y agasajados en el Club Alemán de Barranquilla, donde se llevaron a cabo fiestas como homenaje a los visitantes.

La sociabilidad al interior del grupo de inmigrantes alemanes en Barranquilla también se desarrollaba en el marco de las actividades de otras organizaciones, algunas de ellas relacionadas de manera estrecha con el Nacionalsocialismo, en tanto que otras estaban orientadas hacia actividades culturales típicas de la comunidad de alemanes. Para el primer caso encontramos el Frente Alemán del Trabajo (DAF, por sus siglas en alemán), o mejor, una rama de este movimiento creado en Alemania por los nazis para sustituir a los sindicatos y unificar a los trabajadores dentro y fuera del Reich en un solo colectivo, y que en la ciudad de Barranquilla buscaba captar adeptos entre los inmigrantes germanos cualquiera que fuese su profesión. Para finales de la década del treinta el DAF contaba con instalaciones propias, entre ellas centros de recreo en poblaciones cercanas ubicadas a la orilla del mar, en las que se buscaba que sus asociados se integraran entre sí, al tiempo que descansaban en contacto con la naturaleza ${ }^{44}$.

Para el segundo de los casos mencionados arriba, el de los espacios con una orientación cultural específica, tenemos a la casa Wilhelm Gustloff, que había servido a la comunidad de alemanes en Barranquilla para reunirse alrededor de expresiones artísticas como la poesía, la música y la danza, pero que en la medida en que el Nacionalsocialismo fue cooptando los espacios de reunión de alemanes en la ciudad pasó a ser más un espacio de difusión de las ideas nazis en el que se llevaban a cabo conferencias sobre temáticas como la raza, la cultura alemana (desde un punto de vista nazi) o la política internacional, combinadas con la lectura de los discursos que Hitler daba en Alemania ${ }^{45}$.

La sociabilidad al interior de la comunidad de alemanes en Barranquilla era algo de suma importancia para estos inmigrantes, tanto para ge-

${ }^{44}$ I.A.I, «Unser Badehaus in Puerto Colombia», Karibischer Beobachter, No. 11, 1 de junio de 1939, pp. 12-13.

45 I.A.I, «Wilhelm Gustloff», Karibischer Beobachter, No. 2, 15 de enero de 1937, p. 19. 
nerar cadenas de solidaridad y confianza en un espacio ajeno al propio como para mantener a través del grupo el nexo cultural con su país de origen. Los espacios mencionados en este apartado no fueron otra cosa que la manifestación de ese anhelo de contacto con lo propio, encontrado en los otros de su misma procedencia, con un potencial de cohesión y articulación entre los individuos que terminó por ser objeto de interés de los nazis como una vía para articular a estos alemanes en Barranquilla con el proyecto de la Alemania de Hitler.

Precisamente la llegada a la comunidad de alemanes en Barranquilla de las ideas nacionalsocialistas, con énfasis en las concepciones sobre la diferenciación entre las «razas» y la importancia de mantener la supuesta pureza de sangre germana, podría interpretarse como una situación particularmente crítica para las interacciones que estos inmigrantes europeos mantenían con la sociedad local. Probablemente haya sido así en algunos casos, pero lo que permiten apreciar las fuentes es la existencia de matices, circunstancias más complejas que la simple adopción de las directrices llegadas directamente desde el Reich.

\section{Nacionalsocialismo en Barranquilla: ¿un factor de riesgo para la sociabilidad?}

El 22 de agosto de 1936 se publicó en el magazín nazi que se elaboraba en Barranquilla un articulo en el que se hacía referencia a los antecedentes históricos del Reich que lideraba Hitler. La nota cerraba mencionando que desde el año 936, cuando murió Heinrich I, fundador del primer imperio germano, «la historia del pueblo alemán había sido una constante lucha por mantener la pureza racial, entre otros elementos propios de la germanidad» ${ }^{46}$.

La reafirmación de la especificidad y superioridad racial germana fue uno de los elementos centrales del conjunto de ideas propias del $\mathrm{Na}$ cionalsocialismo que fueron puestas en circulación no sólo en Alemania, sino también fuera del Reich ${ }^{47}$. Para el caso específico de Barranquilla,

46 I.A.I., Olms, «Mil años de Imperio Alemán», Tablón de noticias de los intereses de la comunidad alemana, No. 22, 22 de agosto de 1933, pp.1-3.

47 América Latina no quedó al margen de esta situación, tal y como se expone en una serie de trabajos que abordan el fenómeno de la llegada de ciertas ideas y prácticas propias o estrechamente relacionadas con el Nacionalsocialismo. Para el caso de Chile, por ejem- 
los discursos asociados con el tema de la pureza racial se hicieron particularmente notables en algunas de las publicaciones impresas que editaban los colectivos locales adheridos al Nacionalsocialismo, de manera especial el magazin nazi Karibischer Beobachter, en el que se hacían referencias constantes a la singularidad racial de los alemanes y a la importancia de mantener «limpia» la sangre alemana ante posibles mezclas que terminaran por afectarla ${ }^{48}$.

Este tipo de disposiciones, que la fuerza de las circunstancias hacía de posible cumplimiento en la misma Alemania, resultaban no tan simples de aplicar en una población del Caribe colombiano como Barranquilla. Si bien es cierto que existían espacios de sociabilidad exclusivos para alemanes, mucho más lo es el hecho de que, como ya se mencionó, el éxito económico y social de estos inmigrantes venía dado en buena medida por las relaciones que lograron establecer con la población local, de por sí diversa en su origen, rasgos fenotípicos y cultura, y por el nivel de inserción que habían alcanzado después de décadas de contacto con la sociedad barranquillera.

Ya se hizo mención de casos como el de August Lühr y Hortensia Carbonell, y el de Franz Capell y Raquel Mendoza, parejas colombo-

plo, aparece el estudio de Farías (2000), titulado «Los nazis en Chile»; Ana María Dietrich desarrolló sus estudios doctorales sobre el Partido nazi en Brasil, cuyos resultados se puede encontrar en su tesis alojada en el repositorio institucional de la Universidad de San Pablo; Para el caso de Colombia, es muy conocido el trabajo de Galviz y Donadío (1986), titulado «Colombia Nazi», un estudio de corte periodístico en el que se aborda la presunta actividad de nazis, las respuestas de los organismos de seguridad y el contexto político colombiano de la época. Resulta también de gran interés el análisis más amplio que plantea Max Paul Friedman (2003) sobre la manera como Estados Unidos orientó sus esfuerzos para eliminar la presencia alemana en la región en el contexto de la segunda guerra mundial.

${ }^{48}$ Se hace difícil establecer con precisión la acogida real que las ideas nacionalsocialistas pudieron llegar a tener entre el conjunto de alemanes en Barranquilla. Testimonios de descendientes de algunos alemanes hacen referencia a que una parte de estos extranjeros eran abiertos simpatizantes del nazismo, en tanto que otros optaban por mantener una actitud de reserva, por lo menos parcial, frente a los procesos políticos en Alemania, aunque, eso sí, para evitar inconvenientes con los representantes consulares y diplomáticos del Reich y los efectos derivados de ello, tomaban parte de las diferentes actividades sociales de las organizaciones nazis. De cualquier forma, testimonios sobre ciertos comportamientos de algunos alemanes, registros fotográficos de la época, contenidos impresos y otros recursos documentales más, dan cuenta de, por lo menos, un entusiasmo por el proyecto político nazi extendido de manera notable entre los alemanes en Barranquilla, aunque con distintos niveles de intensidad y en algunos casos motivados por la necesidad y la conveniencia de estar en buenos términos con el gobierno de su país. 
alemanas, que evidencian la situación que se vivía por parte de algunos miembros de la comunidad de alemanes residentes en Barranquilla. Un caso tal vez más complejo fue el de Ella Sálomon, judía alemana que se había radicado en Barranquilla en 1938, y Georg Braun, técnico electricista alemán que vivía en la ciudad caribeña desde 1926 y que trabajaba en una firma cervecera local ${ }^{49}$. No hay evidencias de cercanía de Braun con los círculos sociales alemanes de Barranquilla ni con las organizaciones nazis que operaban en la ciudad, lo que probablemente mantuvo a cubierto de las críticas y comentarios su relación, aunque también es posible que incluso existiendo alguna forma de señalamiento la pareja no se diera por aludida.

Ya en el ámbito de los negocios se puede mencionar a Walter Barthel y Werner von Griesheim, empresarios alemanes que se encontraban asociados en esa década del treinta con el también alemán, pero judío, Herbert Flesch Dreyfus, en la fábrica de pinturas Ico-Flesch \& Cía., de gran éxito no sólo regional sino también nacional, la cual debió ser cerrada en 1942, no por presiones del gobierno alemán sobre sus súbditos no judíos, sino porque la firma fue incluida en la Lista Negra norteamericana ${ }^{50}$, el documento que señalaba, ya en plena Segunda Guerra Mundial, a aquellas empresas o individuos sobre los cuales recaía la sospecha de colaborar o ser simpatizantes del Eje y sobre los que debía aplicarse una forma de bloqueo económico.

Paralelo a estas situaciones puntuales, que evidencian la continuidad de ciertas lógicas de relaciones sociales que se imponen sobre propósitos importados y ajenos al contexto local, se dieron casos de intentos de control sobre el comportamiento de los alemanes y su relación con la sociedad barranquillera. En esta labor los cuerpos representativos del gobierno alemán y del Partido Nacionalsocialista, como el Consulado o el grupo nazi local, cargaban con la responsabilidad de hacer el seguimiento a ciudadanos alemanes.

Pero las formas de sociabilidad no era lo único que importaba, la cuestión racial recibía particular atención, tal y como lo registra un informe de un exempleado colombiano de la oficina consular alemana en Barranquilla elaborado para la Policía Nacional de Colombia: «Tienen

49 A.G.N./A.M.R.E., Diplomática y Consular, Caja 23, Carpeta 181, «Informe de la Sección de Extranjería e Identificación de la Policía Nacional», 23 de mayo de 1943, folios 83 .

50 Manco y Watnik, 2001, pp. 68-71. 
el sistema de control con respecto a los alemanes, para saber quienes son judíos y quienes son arios. Todo alemán que está en Colombia tiene que firmar tres ejemplares de esta declaración, en que constan sus generalidades, lugar de nacimiento en Alemania, profesión, religión, edad y si es judío o ario o a decir mejor legítimo alemán», señalaba el informe ${ }^{51}$.

El estallido de la Segunda Guerra Mundial significó una profunda conmoción en la forma como se habían venido dando las relaciones entre, por lo menos, una buena parte de los alemanes en Barranquilla y la sociedad de acogida. Es difícil establecer qué sucedió con esos alemanes poco visibles, ubicados en estratos socioeconómicos bajos o por fuera de los círculos más dinámicos de la comunidad alemana, pues dejaron pocos registros. Pero existe otro grupo, mucho más visible, integrado por hombres de negocios, profesores, representantes de firmas comerciales y, en líneas generales, individuos que a través de su articulación con la sociedad local al tiempo que participes de los espacios de sociabilidad exclusivos de la comunidad alemana, eran conocidos e identificados en la sociedad barranquillera de la época.

La agresiva política de expansión emprendida por Hitler en Europa hizo que algunos de los ciudadanos de países afectados residentes en Barranquilla tomaran distancia con respecto a estos alemanes en la ciudad. Posteriormente, hacia 1941, con la publicación de la Lista Negra ${ }^{52}$, la situación se hizo todavía más compleja. Tanto para algunos otrora abiertos germanófilos de entre la población local como para quienes la proximidad con alemanes era un asunto dado por temas de negocios, aparecía

51 A.G.N./A.M.R.E., Diplomática y Consular, Caja 21, Carpeta 171: Actividades nazis, «Informe de Manuel Manjarres, ex empleado del Consulado Alemán en Barranquilla, al Ministerio de Relaciones Exteriores», 7 de octubre de 1940, folio 16.

52 La Lista Negra fue un documento emitido en el año de 1941 por parte del Gobierno de los Estados Unidos, en el que aparecían los nombres de ciudadanos y empresas de los países del Eje residentes u operando en América Latina sobre los que recaía la sospecha de que con sus actividades contribuían con el esfuerzo de guerra de sus países de origen. En el documento también aparecían los nombres de ciudadanos o empresas americanos que por sostener algún tipo de cercanía con los previamente incluidos del Eje, pasaban a formar parte de la lista. El efecto de dicho documento era prohibir a las empresas norteamericanas cualquier contacto con los «enlistados», lo que teniendo en cuenta el poder económico de Estados Unidos en la región, fortalecido en tiempos de guerra, representaba un aislamiento difícil de soportar para cualquier empresa o individuo. De esta forma se buscó tender una especie de cerco en torno a los enlistados que afectaría su funcionamiento hasta hacer imposible la continuidad de sus actividades económicas. 
como altamente inconveniente que se les relacionara o que se considerara su cercanía con estos extranjeros que por efectos de la guerra aparecían bajo sospecha. De esta forma, la fuerza de las circunstancias en el marco de la Segunda Guerra Mundial, determinó un antes y un después en la manera en que los alemanes se habían relacionado con la sociedad local. La situación internacional a la que podría considerarse en los años veinte como un componente de gran relevancia en el marco comunitario de migración se presentaba ahora, en el contexto de la guerra como un factor determinante del final, por lo menos temporal, de las relaciones de los alemanes en Barranquilla con la sociedad de acogida.

\section{Conclusión}

La situación de aislamiento a que fue sometida una buena parte de la comunidad de inmigrantes alemanes en Barranquilla durante la segunda guerra mundial, que precedió a la repatriación de un buen número de ellos y a la reubicación de otros más, todo como efecto de las medidas tomadas por el gobierno colombiano en el marco de las disposiciones de seguridad emanadas desde Estados Unidos, constituyó el reverso de la experiencia de vida previa de estos inmigrantes en el Caribe colombiano.

En la medida en que la guerra avanzaba la situación de estos inmigrantes se hizo cada vez más complicada. Atrás quedaron las épocas de sólido contacto con la sociedad local a partir del cual en buena medida se levantó el éxito de muchas de sus iniciativas empresariales y proyectos individuales. En ese nuevo y complicado contexto que trajo el conflicto mundial tampoco fue posible la continuidad de esos espacios de sociabilidad, como el Club Alemán. La instrumentalización que de los mismos hizo el Nacionalsocialismo, sobre todo en la segunda mitad de la década del treinta, terminó siendo uno de entre varios argumentos utilizados luego, ya iniciada la guerra, por los organismos de seguridad nacionales y extranjeros en contra de la permanencia en activo de estos espacios, todo lo cual terminó por hacer de Barranquilla en particular y de Colombia en general unos lugares cada vez menos apacibles y amables para los alemanes $^{53}$.

53 Biermann, 2001. 
Y es que la influencia que el Nacionalsocialismo había venido ejerciendo sobre los alemanes en Barranquilla desde mediados de los años treinta no parece haber tenido la suficiente fuerza y arraigo como para generar una profunda transformación en las formas en que los alemanes se relacionaban con la sociedad de acogida. En un contexto como el Caribe colombiano la adopción irrestricta de comportamientos orientados por ideas de segregación racial habría traído más problemas que beneficios, sobre todo para un grupo de extranjeros cuya economía se basaba en el intercambio comercial y el contacto con una amplia diversidad de actores del ámbito de los negocios. Fueron las disposiciones en materia de seguridad propias de los tiempos de guerra, impulsadas por Estados Unidos y adoptadas por Colombia, las que fragmentaron las redes y prácticas de sociabilidad entre los mencionados extranjeros y la sociedad local.

En la comprensión de estas nuevas circunstancias a que se debieron someter los inmigrantes alemanes en Barranquilla los intereses de la potencia norteamericana resultan particularmente relevantes, puesto que más allá de las consideraciones a que dio lugar la situación de conflicto internacional, un verdadero riesgo para la seguridad de las naciones, que hace entendibles las medidas adoptadas sobre los ciudadanos del Eje, la guerra resultó un escenario adecuado para contrarrestar cualquier forma de influencia extranjera en América Latina que pudiera llegar a competir con las iniciativas de empresarios y empresas de Estados Unidos, y en general con la influencia del país de norte. No sería correcto considerar al contexto de guerra como una simple puesta en escena poco realista de Estados Unidos para consolidar su poder en América Latina, pero tampoco se puede pasar por alto que fue un momento particularmente favorable para incrementar su influencia en la región. Los alemanes en Barranquilla, como en otros lugares del continente americano, lo sintieron de manera especial.

\section{Archivos}

Archivo Histórico del Atlántico (A.H.A.).

Archivo General de la Nación/Archivo Ministerio de Relaciones Exteriores (A.G.N./A.M.R.E.).

Ibero-Amerikanisches Institut (I.A.I) (Instituto Iberoamericano de Berlín). 


\section{Bibliografía}

BIERMANN, Enrique, Distantes y Distintos: los emigrantes alemanes en Colombia, 1939-1945, Universidad Nacional de Colombia, Bogotá, 2003.

BOY, Herbert, Una historia con alas, Editorial Iqueima, Bogotá, 1963.

DALLAS, Gregor, 1918. War and Peace, Overlook Press (Edición para Kindle), S.L., 2001.

DE BEDOUT, Félix, «El pequeños boticario que se volvió gigante», en La presencia alemana en Colombia, AA.VV., Mayr y Cabal, Santafé de Bogotá, 1993.

DIETRICH, Ana María, «Nazismo Tropical? O partido nazista no Brasil», Tesis Doctoral, Universidad de Sao Paulo, 2007. http://www.teses.usp.br/teses/ disponiveis/8/8138/tde-10072007-113709/es.php [Consultado 15 de enero de 2018].

FARIAS, Víctor, Los nazis en Chile, Editorial Seix Barral, Barcelona, 2000.

FLÓREZ, Iveth y LLANOS, José Ramón, Barranquilla y Sabanilla durante el siglo XIX, Ediciones Clio Caribe, Barranquilla, 1995.

FRIEDMAN, Max Paul, Nazis y Buenos Vecinos. La campaña de EE.UU. contra los alemanes de América Latina durante la II Guerra Mundial, Cambridge University Press, Cambridge-New York, 2003.

GALVIZ, Silvia y DONADIO, Alberto, Colombia Nazi, 1939-1945, Planeta, Bogotá D.E., 1986.

GARCÍA ESTRADA, Rodrigo, «Los alemanes en la historia de Antioquia (18501945)», en La presencia alemana en Colombia, AA.VV., Mayr y Cabal, Santafé de Bogotá, 1993.

GÄRTNER, Álvaro, «Las huellas de oro de los mineros alemanes», en La presencia alemana en Colombia, AA.VV., Mayr y Cabal, Santafé de Bogotá, 1993.

HERING, Max. 1892: un año insignificante. Orden policial y desorden social en la Bogotá de fin de siglo, Planeta Colombiana, Bogotá, 2018.

JIMÉNEZ ROMERO, Carlos, ¿Qué es la inmigración?, RBA Libros, Barcelona, 2006.

KITSON, Alison, Germany, 1858-1990: Hope, Terror and Revival, Oxford University Press, Oxford, 2001.

LÁZARO, Julián, «Presencia extranjera en Barranquilla: el caso de los alemanes, sus actividades económicas y el final de su influencia en la urbe caribeña, 1930-1941», Memorias, No. 16. 165-196.

LÁZARO, Julián Andrés, «SCADTA: El viejo anhelo de conquistar el cielo», en Historia Empresarial de Barranquilla (1880-1980), FERRO BAYONA, Jesús, IBARRA, Octavio y GÓMEZ, Eduardo (eds.), Ediciones Uninorte, Barranquilla, 2014, pp. 183-224. 
LOBO, Severino y LOBER-SIES, Dieter, Historia del Colegio Alemán, 1912/131994. Traducción al español: Dörthe de Uribe, Editorial Mejoras, Barranquilla, 1994.

MANCO, Dino y WATNIK, José, Vidas destacadas, Ed. Mancomunicaciones, Barranquilla, 2001.

MARTÍNEZ REY, José. «Bavaria y la industria cervecera colombiana», en $L a$ presencia alemana en Colombia, AA.VV., Mayr y Cabal, Santafé de Bogotá, 1993.

MEISEL, Adolfo y VILORIA, Joaquín, «Los alemanes en el Caribe colombiano: el caso de Adolf Held, 1880-1927», Boletín Cultural y Bibliográfico, Vol. 35, No. 49,1999, 49-83.

NICHOLLS, Theodore, Tres puertos de Colombia: estudio sobre el desarrollo de Cartagena, Santa Marta y Barranquilla, Banco Popular, Bogotá, 1973.

PINZÓN FORERO, Alfonso, La colonización militar y el conflicto colombo-peruano, Asociación Colombiana de Oficiales en de las Fuerzas Militares en Retiro, Bogotá, 1990.

PEUKERT, Detlev, The Weimar Republic: The Crisis of Classical Modernity, Hill and Wang, Nueva York, 1989.

POSADA CARBÓ, Eduardo, El Caribe colombiano. Una historia regional (1870-1950), Banco de la República/ El Áncora Editores, Bogotá, 1998.

POSADA CARBÓ, Eduardo, Una invitación a la historia de Barranquilla, Cámara de Comercio, Barranquilla, 1987.

RODRÍGUEZ PLATA, Horacio, «La inmigración alemana a Santander en el siglo XIX», en La presencia alemana en Colombia, AA.VV., Mayr y Cabal, Santafé de Bogotá, 1993.

SÁNCHEZ, Jesús Paulino El Tesoro de Ambrosio Alfinger, Editorial Gutenberg, Ocaña, 1999.

SOLANO, Sergio y CONDE, Jorge, Elite empresarial y desarrollo industrial en Barranquilla 1875-1930, Ediciones Uniatlántico, Barranquilla, 1993.

VERA DE FLACHS, María Cristina, «Emigraciones transoceánicas. Los alemanes en América. 1850-1914. El caso argentino», Cuadernos de Historia Contemporánea Vol. 16, 1994.

VILORIA DE LA HOZ, Joaquín. Empresarios del Caribe colombiano: historia económica y empresarial del Magdalena Grande y del Bajo Magdalena, 1870-1930, Banco de la República, Bogotá, 2014.

VOS OBESO, Rafaela, Mujer, cultura y sociedad en Barranquilla 1900-1930, Ed. Gente Nueva Ltda, Bogotá, 1999.

WASHAUSEN, Helmut, Hamburg und die kolonialpolitik des Deutschen Reich 1880 bis 1890, Christians, Hamburg, 1968.

WEITZ, Eric, La Alemania de Weimar. Presagio y Tragedia, Turner, Madrid, 2009. 


\section{Datos del autor}

Julián Andrés Lázaro Montes (julianandreslazaro@gmail.com). Doctor en Europa, el Mundo Mediterráneo y su Difusión Atlántica (Universidad Pablo de Olavide, Sevilla); Magister en Ciencias Históricas (Universidad Rey Juan Carlos, Madrid) y Magister en Seguridad, Defensa y Geoestrategia (Campus Internacional para la Seguridad y la Defensa) Universidad a Distancia de Madrid). Actualmente ejerce como Investigador del Instituto Internacional de Estudios del Caribe, de la Universidad de Cartagena. Ha sido docente en pregrado y posgrado en la Universidad del Norte, en asignaturas como Historia Contemporánea, Historia de Colombia e Historia de América Latina y en la Maestría en Historia. También ha sido Director del Archivo Histórico Departamental, en la ciudad de Barranquilla. Ejerció entre 2011 y 2017 como Orientador de Defensa en Armada Nacional de Colombia. 